\title{
Implications of OPV-2 Strain Presence in India \& Strategy to Control the Chance of Endemicity
}

\author{
Dr. Edwin Dias* \\ Professor and HOD, Department of Pediatrics, Srinivas Institute of Medical Science and \\ Research Centre, Mangalore, India \\ *Associate Chief Editor of the Journal \\ E-Mail: dredwindias@gmail.com
}

Type of the Paper: Editorial.

Type of Review: Peer Reviewed.

Indexed In: OpenAIRE.

DOI: http://doi.org/10.5281/zenodo.1471756.

Google Scholar Citation: IJHSP

\section{How to Cite this Paper:}

Dias, Edwin. (2018). Implications of OPV-2 Strain Presence in India \& Strategy to Control the Chance of Endemicity. International Journal of Health Sciences and Pharmacy (IJHSP), 2(2), E1-E4. DOI: http://doi.org/10.5281/zenodo.1471756.

International Journal of Health Sciences and Pharmacy (IJHSP)

A Refereed International Journal of Srinivas University, India.

(C) With Authors

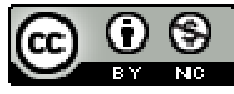

This work is licensed under a Creative Commons Attribution-Non Commercial 4.0 International License subject to proper citation to the publication source of the work.

Disclaimer: The scholarly papers as reviewed and published by the Srinivas Publications (S.P.), India are the views and opinions of their respective authors and are not the views or opinions of the SP. The SP disclaims of any harm or loss caused due to the published content to any party. 


\title{
Implications of OPV-2 Strain Presence in India \& Strategy to Control the Chance of Endemicity
}

\author{
Dr. Edwin Dias* \\ Professor and HOD, Department of Pediatrics, Srinivas Institute of Medical Science and \\ Research Centre, Mangalore, India \\ *Associate Chief Editor of the Journal \\ E-Mail :dredwindias@gmail.com
}

\section{EDITORIAL}

On extensive review and synthesis of vaccine trial data \& community epidemiological studies. In high transmission rates settings, falling population immunity after OPV cessation will lead to conditions in which OPV \& WPVS are capable of causing outbreaks [1]. WHO has officially removed India from the list of countries considered to have never interrupted the transmitted. India has not had a case of polio since 13, January 2011 [2]. India was certified polio free in March 2014 and continues to be so, India continues to remain vigilant against three type of poliovirus. The last polio case due to wide poliovirus in the country was detected on 13 January 2011 [3].

Recently contamination of bivalent oral Polio vaccine, (bOPV) vials has been reported from Uttar-Pradesh. Polio vaccine virus (sabine) type 2 was found in a stool sample of the vaccine in UP who received OPV vaccine supplied by BIOMED. The AFP surveillance system picked this virus. The bOPV vaccine must contain only type 1 and type3sabine polio strains. The use of type 2 poliovirus has been discontinued since this April 2016 and no vaccine should now contain Type2 polio strain (sabine) Recently, type2 polio vaccine virus was found in some sewage and in the stool sample. The detection of type2 vaccine virus indicates that a very robust polio surveillance jointly managed by MOHFW and WHO is still maintained even after seven years have elapsed since the last wild poliovirus case in the country was reported. This detection indicates the use of type 2 poliovirus containing vaccine, despite the fact that OPV contained type 2 poliovirus vaccine had been passed out the gallery and in India in April 2016 as the part of polio endgame strategy.

India has continued WHO engagement to support India maintain the international quality of surveillance of poliovirus detection in the country as a part of intensive surveillance. Sewage collected from the 45 sites and tested for poliovirus in eight accredited polio laboratories in the country. This is in addition to stool samples collected from all individuals, that reports sudden onset of paralysis or polio like symptoms. Every year nearly eighty thousand samples are collected and tested in India.

As a part of an effort to maintain high immunity against all poliovirus, India provides IPV and bOPV to all infants across the country. It is pertaining to note that children over two years of age received tri-valent OPV in routine immunisation till April 2016, when the use of tri-valent OPV was discontinued and bOPV was introduced. Children below two years of age now received bOPV along with IPV or two doses of intradermal fIPV in government immunisation programme and several doses of IPV combination by private providers. The coverage of eligible population of IPV is very good in India, thus population immunity against type 2 polioviruses robust and unlikely event of the spread of type2 strain in the community can hardly cause any harm, further the polio surveillance system in India is very strong and sensitive to pick up the spread of poliovirus. Effective campaign has been undertaken national wide and also sub-national pulse campaign has already been conducted in 201 locations. 
In view of high routine immunisation coverage being achieved in India and admitted of tOPV till 2016, our population level immunity against all three type is very high. This fact was acknowledged and appreciated in the recently conducted study by an Indian expert advisory group on polio comprises of both international and national experts. India has also made remarkable progress under swatch Bharat Abhiyan. A team of MOHFW, DCG1, ICMR, and WHO is continuously monitoring the situation. The government of India is committed to ensuring that all vaccine used in the programme is safe and effective.

One of the main reasons was low vaccine efficacy of tOPV against WPV type1 and type3. VE of tOPV was highest for type2 and WPV of type2 was eliminated in 1999 in the certain state of India, as average per capita vaccine coverage reached. The VE against vaccine type1 and type3 was lowest in Uttar Pradesh and Bihar. Where the force of transmission of WPV was maximum on account of highest infant population density. Transmission was finally interrupted with sustained and extraordinary effort using government machinery and privatepublic partnership. During the year 2004 annual pulse polio vaccination campaigns were conducted 10times each year. Virtually every child was tracked and vaccinated. Including in all transit points and transport vehicles. Since April $25^{\text {th }} 2016$ monovalent OPV types 1 \& 3 was licensed and applied in titrated campaigns according to WPV epidemiology and bivalent OPV (bOPV with both type 1 \& 3) was developed and judiciously deployed. Elimination of WPVS with OPV is only in phase 2 with the introduction of inactivated poliovirus vaccine, switch from tOPV to bOPV and final elimination of all vaccine -derived polioviruses. True polio eradication demands Zero incidences of polio infection, wild\& vaccine. It is my privilege to see the contribution of India in eradicating polio and it remains for the US to eliminate all risk of polio due to wild polio, vaccine virus, including vaccine - derived virus. In my experience, the clinical spectrum of poliomyelitis in India during 1982 to 1993 was bulbar poliomyelitis and many varied presentations of wild virus, paralytic poliomyelitis requiring a lot of clinical support and rehabilitation. In 1952 there were twenty thousand cases of paralytic polio present worldwide.

Both vaccines IPV and bOPV are safe and their use should be continued. India immunisation programme effort for the eradication of poliomyelitis is held in high esteem all over the world. Compared with 2010, WPV cases decreased in India (98\%) in 2011 [4]. Global polio eradication initiative (GPEI), has overcome operational and biological challenges for more complex and daunting than originally envisioned and the paralytic poliomyelitis cases have fallen from $\sim 350,000$ in 1988 to 22 in 2017 [5]. True polio eradication demands Zero incidences of polio infection, wild \& vaccine. It is my privilege to see the contribution of India in eradicating polio and it remains for us to eliminate all risks of polio due to wild polio, vaccine virus, including vaccine - derived virus. The key to focusing and preventing next poliomyelitis (OPV2) epidemic is to understand mechanism underlying disease emergence. Strategies to deal with this issue proactively will require along with increased public health financial investment multidisciplinary efforts along with surveillance infrastructure and sentinel paediatrician and public health official training. An important strategy to contain type 2 OPVIRUS would be to do serological estimation of antibodies to OPV 2 strain for those receiving intradermal IPV, 2 DOSES as per GOVERNMENTAL NOTIFICATION and then modifying the IPV SCHEDULE thereafter to prevent an OPV 2 HYPERENDEMIC situation in India.

\section{REFERENCE:}

[1] Famulare M, Slinger C, McCarthy KA, Eckhoff PA, Chabot - coture G (2018) assessing the stability of polio eradication after the withdrawal of oral Polio vaccine. PLoSbiol 16(4) ; e2002468. DOI: http://doi.org/10.1371/journal.pbio.2002468. 
[2] India officially removed from the list of countries polio endemic countries. (accessed on July 25,2012). Available from: http://new.paho.org/hq/index.php?option=com_cont ent\&task=view\&id=6484\&lt.

[3] Bahl S, Kumar R, Menabde N etal, Polio-free certification and lessons learned - - southEast Asia region, March 2014. MMWR Morgan Mortal Wkly Rep. 2014 October 24;63(42):941-6.

[4] CDC. Progress towards interruption of wild poliovirus transmission - worldwide, January 2010-March 2011. MMWR 2011; 60:582-6.

[5] Kew O. Reaching the last one percent : Progress and challenges in global polio eradication. Curr Opin virol. 2012;2:2188-98 (PubMed). 